\title{
Wave characteristics estimation by GPS receivers installed on a sailboat travelling off-shore
}

\author{
Paolo De Girolamo \\ Department of Civil, Building and \\ Environmental Engineering \\ Sapienza University of Rome \\ Rome, Italy \\ paolo.degirolamo@uniroma1.it
}

\author{
Augusto Mazzoni \\ Department of Civil, Building and \\ Environmental Engineering \\ Sapienza University of Rome \\ Rome, Italy \\ augusto.mazzoni@uniroma1.it
}

\author{
Mattia Crespi \\ Department of Civil, Building and \\ Environmental Engineering \\ Sapienza University of Rome \\ Rome, Italy \\ mattia.crespi@uniroma1.it
}

\author{
Marcello Di Risio \\ Department of Civil, \\ Construction-Architectural \\ and Environmental Engineering \\ Environmental and Maritime Hydraulic \\ Laboratory (LIam) \\ University of L'Aquila \\ L'Aquila, Italy \\ marcello.dirisio@univaq.it
}

\author{
Myrta Castellino \\ Department of Civil, Building and \\ Environmental Engineering \\ Sapienza University of Rome \\ Rome, Italy \\ myrta.castellino@uniroma1.it
}

\author{
Alessandro Romano \\ Department of Civil, Building and \\ Environmental Engineering \\ Sapienza University of Rome \\ Rome, Italy \\ alessandro.romano@uniroma1.it
}

\author{
Department of Engineering \\ Rome, Italy \\ giorgio.bellotti@uniroma3.it
}

\author{
Davide Pasquali \\ Department of Civil, \\ Construction-Architectural \\ and Environmental Engineering \\ Environmental and Maritime Hydraulic \\ Laboratory (LIam) \\ University of L'Aquila \\ L'Aquila, Italy \\ davide.pasquali@univaq.it \\ Paolo Sammarco \\ Department of Civil and Computer \\ Engineering \\ University of Rome "Tor Vergata" \\ Rome, Italy \\ sammarco@ing.uniroma2.it
}

\begin{abstract}
The paper describes a measurement campaign carried out by means of GPS receivers installed on board of a moving sailboat and the application for the estimation of the local wave properties. The movements of the boat, as reconstructed using the present approach, may be used for many useful practical purposes, as for example the improvement of autopilots or for real time control systems of ships, to analyze and improve the performance of race sailboats, to estimate the local properties of the waves. An application of the method to estimate the wave properties during a storm in the Western Mediterranean Sea is presented, and the results compared against two sets of hindcast data and wave buoy records.
\end{abstract}

Keywords - Ship motions, wave characteristics, GPS data analysis, sailboat, off-shore

\section{INTRODUCTION}

Estimation of ship motions and wave properties from a travelling ship is of utmost importance for many ocean engineering applications [13], e.g.: (i) to improve the performance of autopilots and control systems of ships [1] or in general of floating structures [19]; (ii) to provide real-time wave data for Global Forecasting Systems (GFS) numerical models and for the calibration/validation of wave forecasting/hindcasting models (e.g. [7]), etc.; (iii) to analyze the performance of a race sailboat, i.e. to derive actual velocity polar curves in the presence of waves and to correct wind measurements carried out onboard.

As far as the estimation of the wave properties is concerned, a possible approach is to derive the parameters of interest directly on the basis of the ship motion, taking into account the dynamic ship frequency response function $[10,12,13,20]$.

In the last fifteen years the measurement of directional wave spectra on the basis of moving ships kinematic has been extensively studied. Some approximated solutions of the main theoretical problem arising from the transformation of encounter frequencies measured onboard into the true wave frequencies have been derived. This problem is known as the "triple-valued function problem" which is due to the non-linear Doppler shift effect induced by frequency dispersive waves. Approximated solutions have been obtained by using both parametric methods, which assume the shape of the wave spectrum, and non-parametric (stochastic) methods, where the spectrum shape is not prescribed a priori $[13,18]$.

On-board measurements of ship-motion is normally carried out by means of the same sensors used for the pitch/roll accelerometric directional wave buoys [14,15,17]. Only in the last ten years, the GPS technology has been applied on floating buoys for measuring directional wave spectra $[8,9]$. The advantages, as well as the disadvantages, of using GPS buoys are pointed out by [9].

The present paper describes the computation of ship motion using GPS receivers, installed on a travelling sailboat and the application of the ship motion for the computation of the local wave properties. The considered sailboat is a small oceanic race boat (LOA of $12,0 \mathrm{~m}$ ) characterized by a very light displacement. She is a Class 40 oceanic vessel named ECO40, which left from the port of "Riva di Traiano" located close to Rome (Italy) on October 19th, 2014 to perform a non-stop sailing alone around the World in energy 


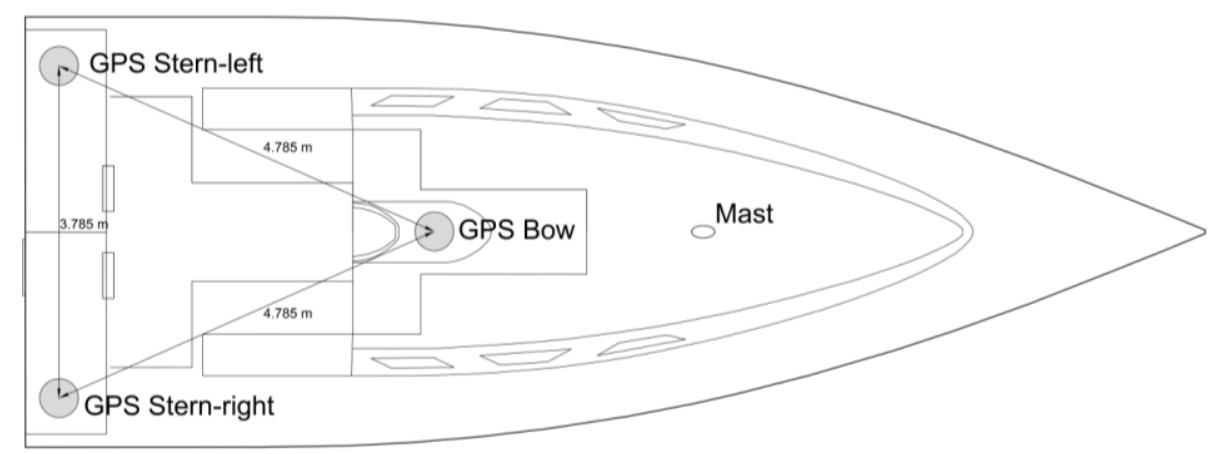

Fig. 1. Plan view of the sailboat ECO 40 and of the three GPS antennas.

and food self-sufficiency. The boat route goes through the Gibraltar Strait, then descends the Atlantic Ocean and sailing around the Antarctic, at a mean latitude of $500 \mathrm{~S}$, from West to East, rounding the famous capes of the world: Cape of Good Hope, Cape Leeuwin and Cape Horn. Finally, ascending the Atlantic Ocean and passing again the Strait of Gibraltar coming back to homeport [5,6].

The boat was equipped with three high precision GPS receivers (rover), provided by Leica Geosystem, for measuring the movements of the boat. During offshore oceanic sailing the GPS measurements cannot be corrected using on land reference receivers, or a GNSS (Global Navigation Satellite System) network, since the distance between rovers and reference station should not be larger than few tens of kilometers. In order to solve this problem, in the present work two GPS data processing techniques have been complementarily applied, by using an innovative approach. The processed data provided by the GPS receivers have been used to compute the boat motion and the boat motion applied to estimate the waves properties faced by the sailboat during its navigation around the world.

In the following sections a synthetic description of the work is illustrated.

\section{METHODS}

ECO40 was equipped with three geodetic class GPS antenna/receiver systems provided by Leica Geosystems AG, able to acquire code and phase observations on both L1 and L2 frequencies. The GPS antennas have been installed on board ECO40 as shown in Fig. 1. Two antennas has been mounted on the stern roll bar (they are indicated as GPS Stern-right and GPS Stern-left in the figure) and one antenna (GPS Bow), was installed along the main axis of the boat, close to the entrance and protected by a small fiberglass structure. The positions of the three GPS antenna are at the vertices of an isosceles triangle as described in the Fig. 1.

The GPS raw code and phase observations on both L1 and L2 frequencies were acquired by each above described GPS antenna/receiver systems (in the following indicated as "receiver") with a sampling rate of $2 \mathrm{~Hz}$. The raw observations have been stored on a flash-card by each receiver. The data analysis was carried out in post-processing after the recovery of the flash-cards. The analysis carried out in the post processing described in the following, may be however carried out in real time onboard of the sailboat. The post-processing was carried out by employing two different techniques: the variometric approach and the moving base kinematic.

The Variometric Approach for Displacements Analysis Standalone En- gine (VADASE) is an innovative GPS data processing approach proposed in the recent past [2,3]. The approach is based on time single differences of carrier phase observations continuously collected using a standalone GPS receiver and on standard GPS broadcast products (orbits and clocks) that are available in real time. Therefore, one receiver works in standalone mode and the epoch-by-epoch displacements (equivalent to velocities) are estimated. In this work, a kinematic extension of the variometric approach (Kin-VADASE) developed specifically for the navigation field was used.

A second approach was used. Indeed, the most widely used technique in GPS kinematic positioning is based on the using of two devices: the rover moving receiver and a reference static receiver of known coordinates. In order to obtain high accuracy results, the distance between the rover and the reference receiver should not exceed few tens of kilometers. Then it is almost impossible to apply this technique in off-shore navigation, due to the lack of close reference receivers availability. Nevertheless, in this work we present an innovative application of differential kinematic positioning applied to a reference moving (hereinafter referred to as moving base kinematic, MBK). With respect to this reference moving receiver, it was possible to estimate, epoch by epoch, the positions of another receiver. This technique does not allow to define the absolute position of the receiver but only the relative one, which however is very accurate, since the distance between each couple of receivers is known. Then, the method has been applied to three couples of receivers deployed onboard (Fig. 1): GPS Bow GPS Stern-right; GPS Bow - GPS Stern-left and GPS Sternright - GPS Stern-left.

The results of the GPS data post processing were used to reconstruct the $6 \mathrm{DOF}$ boat motion and to estimate the wave parameters encountered by the sailing boat (i.e. significant wave height and mean wave direction).

In order to compute the boat heave, it is necessary to use a technique able to provide the absolute vertical position of the boat. Then, the Kin-VADASE method has been applied by using the $2 \mathrm{~Hz}$ up velocities time history of each receiver. Nevertheless, the up-velocity component provided by KinVADASE method is subjected to bias, mainly related to the 
number of visible satellites and to their position with respect to the GPS antennas location. To eliminate the bias a high pass filter was used. The high pass filter has been applied to the vertical movement time history obtained by integrating over the time the up-velocity component provided by a GPS antenna. From some tests it was seen that for a 30-minute signal the cut-off period of the filter can be taken around 2 minutes (i.e. cut-off frequency $\mathrm{fc}=0.0167 \mathrm{~Hz}$ ). The cut-off frequency has been chosen large enough to avoid the low frequency drift related to the numerical integration, but small enough to analyze both the sea and the swell wave features.

Finally, the boat heave time series has been obtained by computing the instantaneous mean values of the three filtered heave time series as obtained by each of the three GPS antennas. Fig. 2 shows an example of the calculated boat heave signal for a time window of 4 days.

For the computation of the boat roll motion it is not necessary to use a technique able to provide the absolute position of the boat, as roll may be obtained by means of the relative vertical differences between two points located along position of the couple of Stern-GPSs. This technique is very accurate and it is not affected by bias.

The computation of the pitch motion, as for the roll, has been carried out by using the moving base kinematic (MBK) technique. For this parameter, the two GPS receivers located along the main longitudinal axis of the sailboat are not available and therefore a direct estimation of the pitch cannot be obtained. Two couples of GPS receivers have therefore been considered: the bow/stern-right and the bow/stern-left. It has to be stressed that the signals are contaminated by a component of the roll motion, since the two-axis passing for each couple of GPS (bow-stern right and bow-stern left) are not parallel to the main boat axis. In order to obtain the pitch signal depurated by the roll component, a mean signal (pitch) obtained from the two couples bow - stern right and the bow - stern left has been computed.

The yaw angle has been computed by using the MBK technique. The two GPS receivers placed on the stern of the sailboat (stern-right and stern left) have been used to calculate the relative horizontal angle that the main

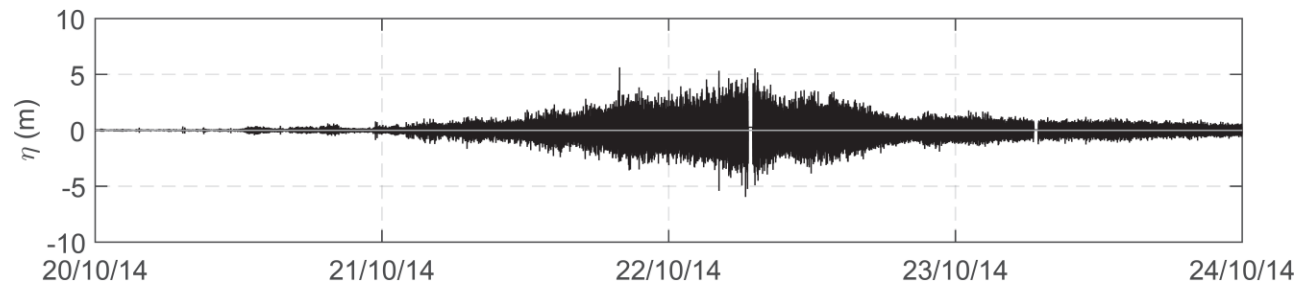

Fig. 2. Example of the boat heave time series over a time window of 4 days, obtained by calculating the instant mean values of the three filtered heave time series as obtained by each GPS antenna.

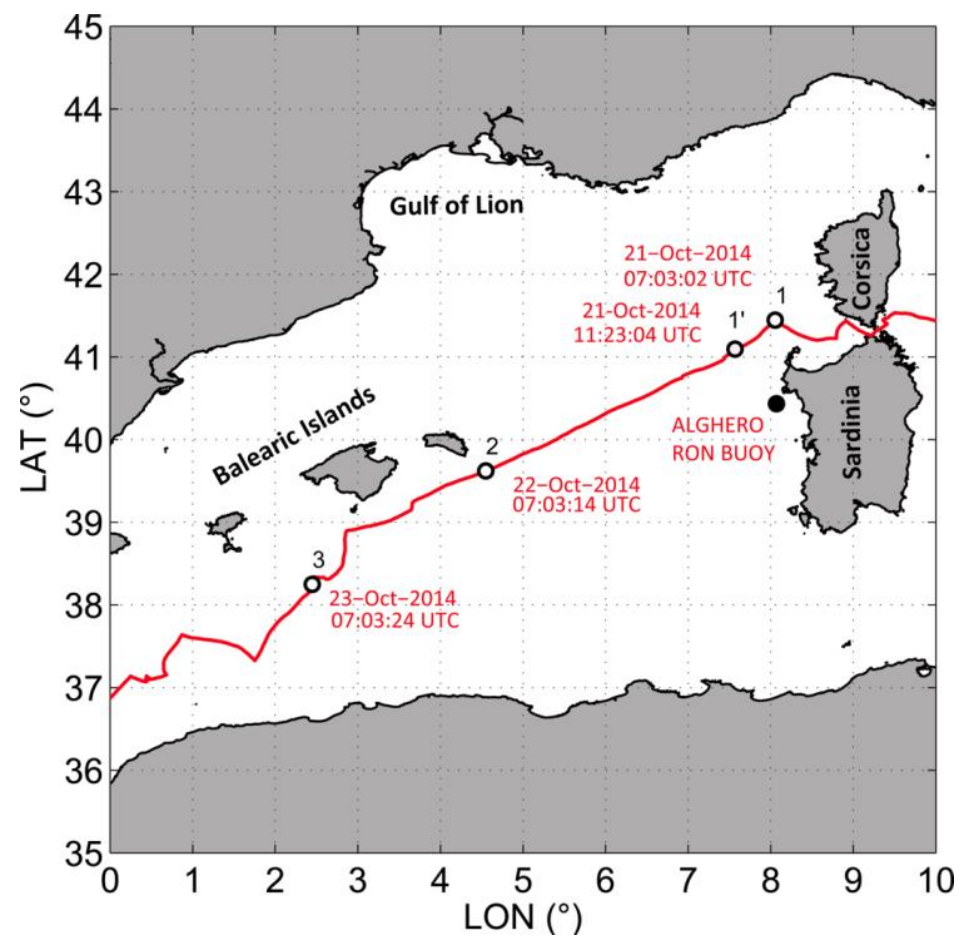

Fig. 3. The route in the western Mediterranean Sea followed by ECO 40. The empty dots refer to four points of interest along the route and the black dot identifies the position of the Alghero wave buoy.

an axis orthogonal to the main longitudinal axis of the boat. Then the roll motion angle has been computed by using the moving base kinematic (MBK) applied to the relative Up longitudinal axis of the sailboat forms with respect to the orientation of the same axis, evaluated at the previous time step. Hence, the yaw motion may be described by means of 
the relative differences of two points located along an axis orthogonal to the main longitudinal axis of the sailboat.

The estimate of the surge and sway motions requires to use the absolute position of the boat in the horizontal plane. Therefore, the Kin-Vadase method has been used again. The North and East velocities components have been processed. It has to be stressed that the horizontal velocities provided by the Kin-Vadase method are much more accurate with respect to the vertical one used for the heave computation. Indeed, starting from a known position and orientation of the boat as obtained by the three GPS receivers, the planimetric error of the method, evaluated over a time window of 30 minutes, is less than 1 meter.

\section{RESULTS}

On October 19th 2014, when ECO40 left from the Italian Port Riva di Traiano (Italy) directed to Gibraltar Strait, the weather forecasts suggested that within the next 24/48 hours, the first seasonal front of cold air was expected to induce Mistral winds with speed exceeding 40 knots, blowing from the Gulf of Lion [5]. ECO40 was able to reach the Asinara typical for standard analysis of the free surface elevation records as measured from standard wave buoys, is considered to be long enough to properly describe, in a statistical sense, the considered sea state and short enough for considering the wave features to be stationary [11].

Fig. 4 shows the significant wave height time series of the storm obtained from the heave signal by using both the zero-crossing analysis $\left(\mathrm{H}_{1 / 3}\right)$ and the frequency spectrum analysis $\left(\mathrm{H}_{\mathrm{m} 0}\right)$ carried out in the encountered frequency domain. The maximum zero crossing wave height $\left(\mathrm{H}_{\max }\right)$ is also represented. The maximum values obtained during the storm for $\mathrm{H}_{1 / 3}$ and for $\mathrm{H}_{\mathrm{m} 0}$ are respectively $5.84 \mathrm{~m}$ and 5.42 $\mathrm{m}$ while $\mathrm{H}_{\max }$ is of $9.6 \mathrm{~m}$. The vertical dashed lines refer to four points of interest along the route (as shown in Fig. 3).

To assess the accuracy of the estimated wave parameters, Fig. 5 shows the comparison between the significant wave height as (i) measured by the Alghero RON wave buoy, (ii) estimated by the ECMWF analysis and (iii) by DICCA for a grid point close to the buoy position. The Alghero wave buoy belongs to the "RON-Rete Ondametrica Nazionale" (Italian Wave Measurements Network, [4]), managed by

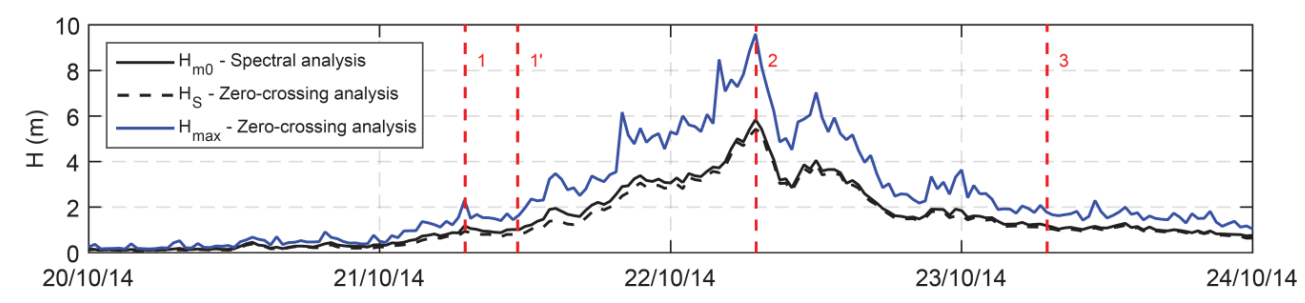

Fig. 4. Significant wave height obtained from the heave by using zero crossing analysis (black dashed line) and frequency spectrum analysis (black line). The maximum wave height is reported as well (blue line).

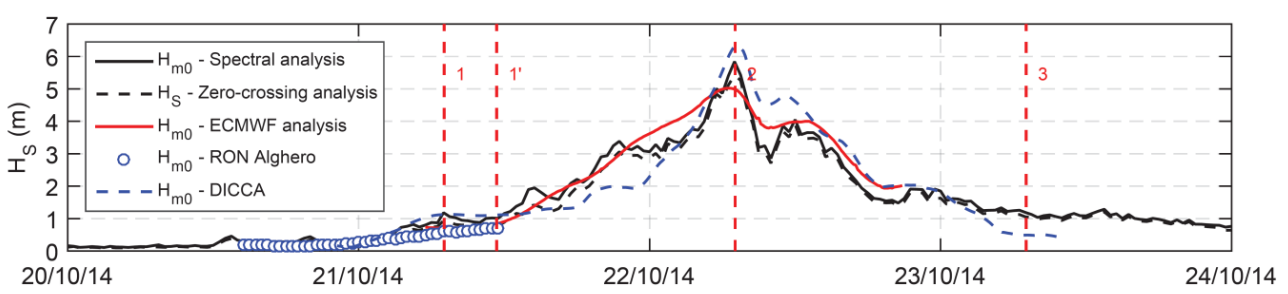

Fig. 5. Comparison between the significant wave height measured onboard and the available wave data.

Island and to follow the route towards the Balearic Islands before the arrival of the main storm: the sailboat has faced the storm running on the quarter. The route between the Asinara Island and the Balearic Islands is represented in Fig. 3. The figure also provides information on the travel times.

The features of the waves faced by the sailboat during the Gulf of Lion event have been derived from the sailboat heave, roll and pitch motions described in the previous section. Since ECO40 may be classified as an ultra-light displacement boat (she has full loaded a displacement of about $4700 \mathrm{~kg}$, a length of about 12,0 $\mathrm{m}$ and a maximum breadth of $4,5 \mathrm{~m}$ ), it has been assumed that the sailboat follows the sea surface, thus assuming a unitary transfer function between the boat and the sea surface movements (i.e. the Response Amplitude Operator is the Unit Matrix).

The heave, pitch and roll signals have been analyzed within time windows of 30 minutes. This duration, which is
ISPRA up to 2014 and now dismissed after about 23 years; the buoy was located offshore the North-West coast of Sardinia and it was exposed to the waves generated by the storm at hand. The six-hourly hindcast wave data provided in analysis by ECMWF (European Centre for Medium-Range Weather Forecasts) and the the hourly wave hindcast data provided by DICCA (Department of Civil, Chemical and Environmental Engineering, University of Genoa, Italy), covering 36 (1979-2014) years over the Mediterranean Sea [6] were also considered. To obtain the waves faced by the sailboat along her route, the ECMWF and DICCA data have been interpolated in time and in space by means of bi-linear technique.

\section{CONCLUDING REMARKS}

A method to calculate the movements of a boat, using three GPS receivers, has been presented. It is based on the combined approach of the kin-Vadase and Moving Base 
Kinematic techniques. Application to the records obtained onboard the ECO 40 sailboat during a storm in the Western Mediterranean Sea has been presented. Wave properties as derived on the basis of the estimated boat movements are compared to available wave data. It appears that the wave properties are calculated with a high degree of accuracy.

In general, the techniques proposed in this paper appear suitable to estimate the 6DOF movements of vessels navigating offshore, where onshore reference receivers or GNSS network cannot be used to correct GPS errors. The motion of the boat can provide input for many important practical applications, such as the improvement of autopilots, the analysis and the optimization of ship performances, and the estimation of local wave properties. Furthermore, it can be used to correct wind measurements carried out onboard sail boats.

\section{ACKNOWLEDGMENTS}

Dr. Pier Paolo Pecoraro (IDRO-GEOTEC) is acknowledged for his help and expertise during the installation of the instruments. The skipper Matteo Miceli is also acknowledged for his contribution. A special acknowledgement is due to Leica Geosystem for providing the high-precision GPS receivers and to Prof. Giovanni Besio for providing the DICCA wave data.

\section{REFERENCES}

[1] Belleter, D. J., Galeazzi, R., Fossen, T. I., 2015. Experimental verification of a global exponential stable nonlinear wave encounter frequency estimator. Ocean Engineering 97, 48-56.

[2] Colosimo, G., Crespi, M., Mazzoni, A., 2011a. Real-time gps seismology with a stand-alone receiver: A preliminary feasibility demonstration. Journal of Geophysical Research: Solid Earth (19782012) 116 (B11).

[3] Colosimo, G., Crespi, M., Mazzoni, A., Dautermann, T., 2011b. Coseismic displacement estimation: Improving tsunami early warning systems. GIM INTERNATIONAL 25, 19-23.

[4] De Boni, M., Cavaleri, L., Rusconi, A., 1992. The Italian waves measurement network. Proceedings of 23rd Conference on Coastal Engineering, Venice, Italy, 1992.

[5] De Girolamo, P., Romano, A., Bellotti, G., Pezzoli, A., Boscolo, A., Crespi, M., Mazzoni, A., Di Risio, M., Pasquali, D., Franco, L., Sammarco, P., 2015. Analysis of the 21/22 october 2014 storm experienced by the sailboat eco40 in the Gulf of Lion. Proceedings of the 3rd International Congress on Sports Sciences Research and Technology Support

[6] De Girolamo, P., Romano, A., Bellotti, G., Pezzoli, A., Castellino, M., Crespi, M., Mazzoni, A., Di Risio, M., Pasquali, D., Franco, L., Sammarco, P. Met-ocean and heeling analysis during the violent $21 / 22$ october 2014 storm faced by the sailboat ECO40 in the gulf of lion: Comparison between measured and numerical wind data (2016) Communications in Computer and Information Science, 632, pp. 86105 .

[7] De Girolamo, P., Di Risio, M., Beltrami, G.M., Bellotti, G., Pasquali, D. The use of wave forecasts for maritime activities safety assessment (2017) Applied Ocean Research, 62, pp. 18-26.

[8] Hauser, D., Kahma, K., Krogstad, H. E., et al., 2005. Measuring and analysing the directional spectra of ocean waves.

[9] Herbers, T., Jessen, P., Janssen, T., Colbert, D., MacMahan, J., 2012. Ob- serving ocean surface waves with gps-tracked buoys. Journal of Atmospheric and Oceanic Technology 29 (7), 944-959

[10] Hirayama, T., 1987. Real-time estimation of sea spectra based on motions of a running ship, 2nd report: Directional wave estimation. Journal of the Kansai Society of Naval Architects (204)
[11] Holthuijsen, H. L., 2010, Waves in Oceanic and Coastal Waters, Cambridge University Press, ISBN: 9780521129954

[12] Iseki, T., Ohtsu, K., Fujino, M., 1992. A study on estimation of directional spectra based on ship motions ii-experimental investigation for accuracy. The Journal of Japan Institute of Navigation

[13] Iseki, T., Ohtsu, K., 2000. Bayesian estimation of directional wave spectra based on ship motions. Control Engineering Practice 8 (2), Tannuri, E. A., Sparano, J. V., Simos, A. N., Da Cruz, J. J., 2003. Estimating directional wave spectrum based on stationary ship motion measurements. Applied Ocean Research 25 (5), 243-261.215-219.

[14] Longuet-Higgins, M. S., Cartwright, D., Smith, N., 1963. Observations of the directional spectrum of sea waves using the motions of a floating buoy

[15] Long, R. B., 1980. The statistical evaluation of directional spectrum esti- mates derived from pitch/roll buoy data. Journal of Physical Oceanogra- phy 10 (6), 944-952

[16] Mentaschi, L., Besio, G., Cassola, F., and Mazzino, A.: Performance evaluation of WavewatchIII in the Mediterranean Sea, OceanModelling, 90, 82-94, 2015.

[17] Mitsuyasu, H., Tasai, F., Suhara, T., Mizuno, S., Ohkusu, M., Honda, T., Rikiishi, K., 1975. Observations of the directional spectrum of ocean wavesusing a cloverleaf buoy. Journal of Physical Oceanography 5 (4), 750-760

[18] Nielsen, U. D., 2006. Estimations of on-site directional wave spectra from measured ship responses. Marine Structures 19 (1), 33-69

[19] Tannuri, E. A., Sparano, J. V., Simos, A. N., Da Cruz, J. J., 2003. Estimating directional wave spectrum based on stationary ship motion measurements. Applied Ocean Research 25 (5), 243-261

[20] Webster, W. C., Dillingham, J. T., 1981. Determination of directional seas from ship motions. Proceedings of Directional Wave Spectra Application 